\title{
Pulmonary Embolism Caused by Intravenous Leiomyosarcoma of the Lower Limb
}

\author{
Soichiro Kado ${ }^{1}$, Masahide Goto ${ }^{1,2}$, Hidetsugu Yamao ${ }^{3}$, Toru Tsukada ${ }^{4}$, \\ Masataka Sato ${ }^{4}$ and Yoshifumi Uekusa ${ }^{1}$
}

\begin{abstract}
:
Pulmonary embolism (PE) is usually caused by thrombosis or tumor. We report the long-term survival of a patient with PE due to a leiomyosarcoma in the deep vein. A 71-year-old woman complained of dyspnea and swelling of the left lower limb. Computed tomography revealed filling defects in the pulmonary arteries and deep vein. She was diagnosed with PE caused by venous thrombosis and treated with anticoagulant therapy. Her symptoms were prolonged, and D-dimer tests remained negative. Biopsy of the substance in the deep vein revealed leiomyosarcoma. The possibility of PE caused by extravascular or intravascular tumors should be considered when a patient is negative for D-dimer.
\end{abstract}

Key words: deep vein thrombosis, venous thromboembolism, leiomyosarcoma, pulmonary embolism

(Intern Med 57: 1425-1428, 2018)

(DOI: 10.2169/internalmedicine.0030-17)

\section{Introduction}

Among elderly patients presenting with dyspnea in outpatient departments, the most common conditions are decompensated heart failure, pneumonia, chronic obstructive pulmonary disease (COPD), asthma, and pulmonary embolism (PE) (1). Exclusion of PE should be especially considered when computed tomography (CT) shows filling defects in the pulmonary artery and deep vein (2). Well's criteria are useful for diagnosing PE (3). If the score is $\leq 4$ points and $\mathrm{D}$-dimer is negative, the probability of $\mathrm{PE}$ is only $2.2 \%$. If the score is $>4$ points and D-dimer is negative, the probability of PE is $<20 \%$. Thus, the measurement of the D-dimer level is very important for the diagnosis of PE. On the contrary, pulmonary tumor embolisms are rare, but can cause dyspnea. The primary tumors that most commonly cause embolisms are breast, lung, and gastric carcinoma (4).

We herein report a case in which the measurement of a patient's D-dimer level led to the diagnosis of PE due to deep intravenous leiomyosarcoma of the lower limb, despite the fact that venous thromboembolism (VTE) was suspected from the $\mathrm{CT}$ findings of filling defects in the pulmonary ar- tery and deep vein.

\section{Case Report}

A 71-year-old Japanese woman presented to our outpatient department with dyspnea and swelling of the left lower limb. She had a history of COPD and hepatitis C. A physical examination revealed a heart rate of 106/min, an oxygen saturation of $96 \%$ (room air), clear chest auscultation, and unilateral swelling of the left leg. Contrast-enhanced CT was performed, which revealed filling defects in the pulmonary artery and deep vein (from the superficial femoral vein to the external iliac vein) of the left lower limb (Fig. 1). There was no swelling in the axillary or inguinal lymph nodes. Vascular ultrasound showed high- and low-density areas in the femoral vein, which were consistent with chronic and acute clots, respectively. A laboratory analysis revealed no evidence of coagulation abnormalities or bacteremia and the patient's D-dimer level was low $(0.6 \mathrm{ng} / \mathrm{mL})$. Despite these findings, she was diagnosed with PE caused by VTE based on a Well's score for PE of 7.5 points, which included tachycardia, clinical signs of PE, and symptoms of DVT. Treatment with anticoagulant therapy was initiated. After 1

${ }^{1}$ Department of Internal Medicine, Kitaibaraki City Hospital, Japan, ${ }^{2}$ Department of Pediatrics, Jichi Medical University, Japan, ${ }^{3}$ Department of Cardiovascular Medicine, Kitaibaraki City Hospital, Japan and ${ }^{4}$ Department of Cardiovascular Surgery, Hitachi General Hospital, Japan Received: August 4, 2017; Accepted: October 10, 2017; Advance Publication by J-STAGE: January 11, 2018 Correspondence to Dr. Masahide Goto, mgoto@jichi.ac.jp 

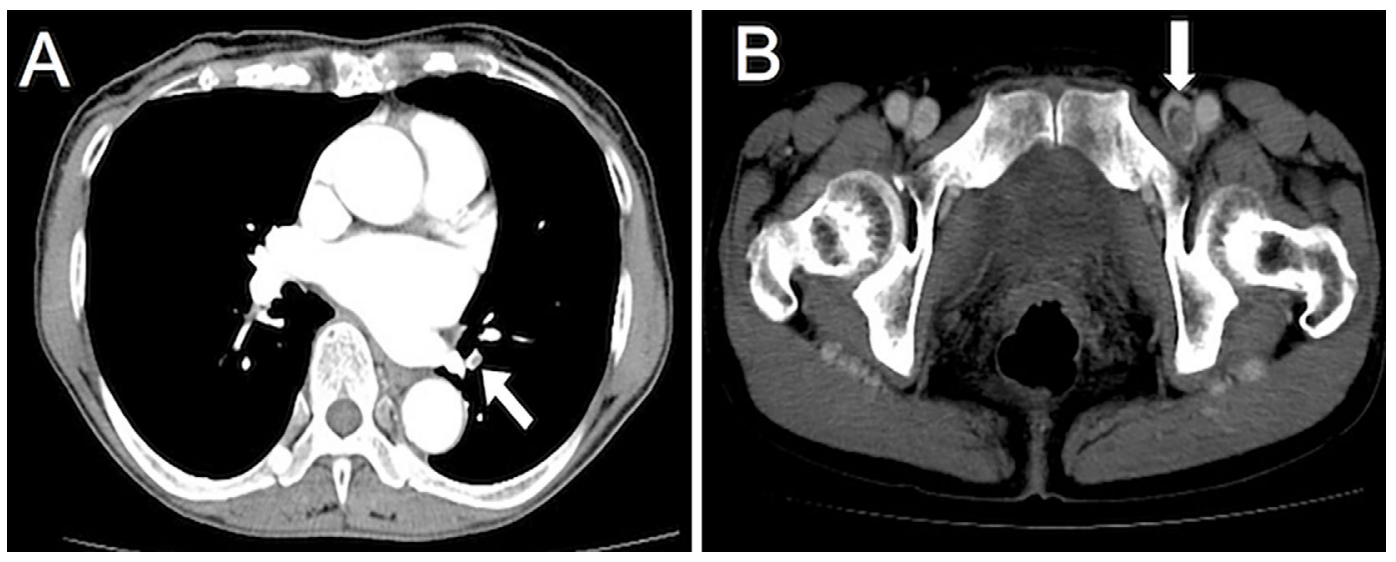

Figure 1. The contrast-enhanced computed tomography (CT) findings: Filling defects in the pulmonary artery (A, arrow) and deep vein of the left lower limb (B, arrow). The mass $(18.5 \times 20.2 \mathrm{~mm})$ in the lower limb was smooth and irregular and seemed to be in contact with the venous walls but had not invaded the surrounding tissue. The venous wall was slightly dilated but not displaced by the masses.
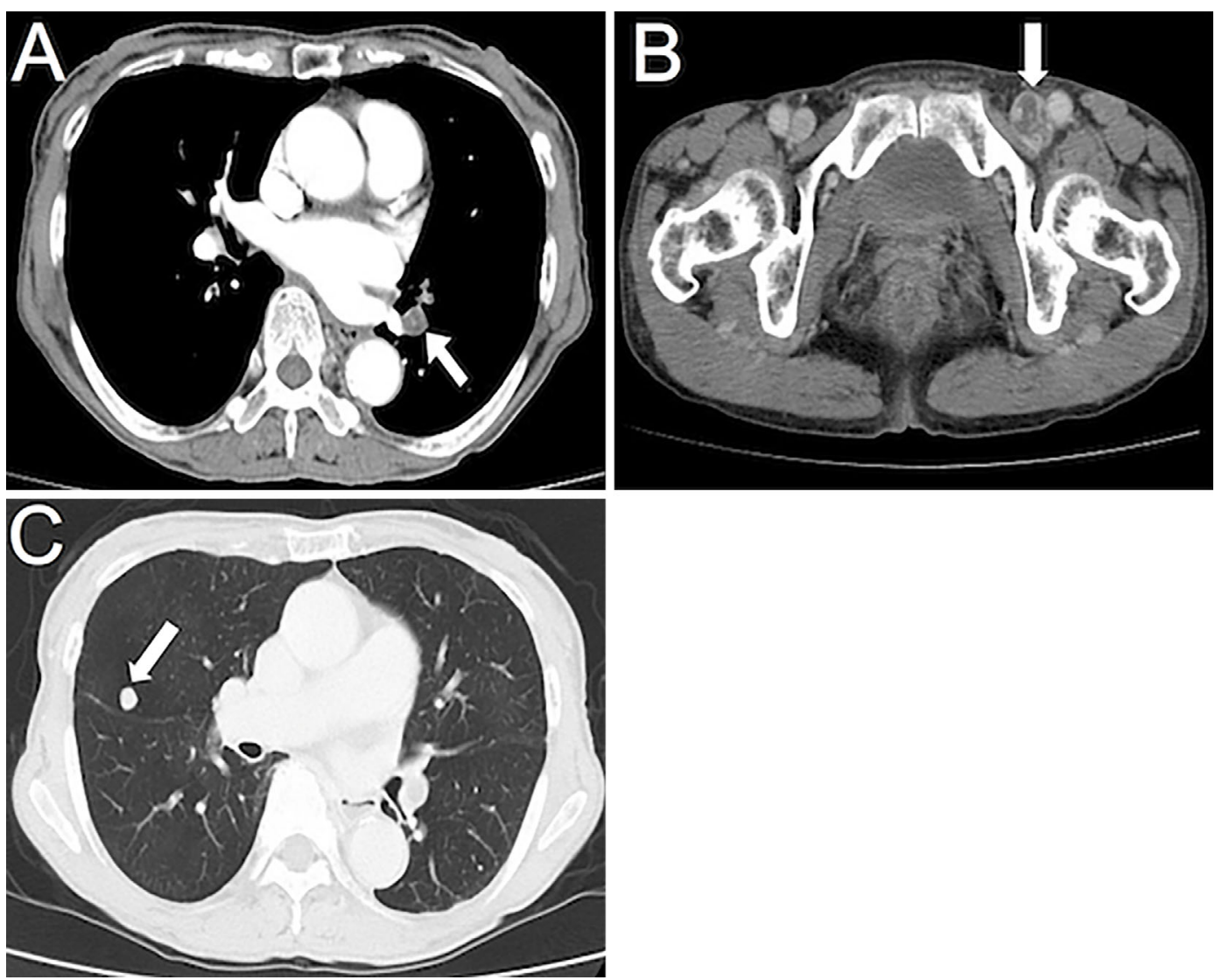

Figure 2. The CT findings 4 months later: Enlargement of the filling defects in the pulmonary artery (A, arrow) and the deep vein of the left lower $\operatorname{limb}(20.3 \times 25.7 \mathrm{~mm})$, which appeared as highdensity areas in the mass $(B$, arrow). A new mass was detected in the right lung $(C$, arrow).

week of anticoagulant therapy, CT revealed that the filling defects in the pulmonary artery and deep vein had not worsened. After 4 months of anticoagulant therapy, the patient's dyspnea and left lower limb swelling persisted, and D-dimer tests remained negative. CT showed the enlargement of the filling defects in the pulmonary artery and deep vein of the lower limb and a new mass in the right lung (Fig. 2). A bi- opsy of the substance in the left superficial femoral vein was performed, based on the suspicion of a malignant tumor. A histological examination revealed that the tumor was composed of spindle cells with atypia (Fig. 3). Immunohistochemical staining demonstrated that the tumor expressed desmin, vimentin, and smooth muscle actin but not cluster of differentiation 31 , cluster of differentiation 34 , stem cell 
growth factor receptor, or mouse double minute 2 homolog. These findings confirmed the diagnosis of leiomyosarcoma. Positron emission tomography detected only one metastatic tumor in the right lung. Accordingly, the clinical stage of the patient was determined to be stage IV. Subsequently, she underwent standard first-line chemotherapy with doxorubicin. The chemotherapy regimen was effective and she was

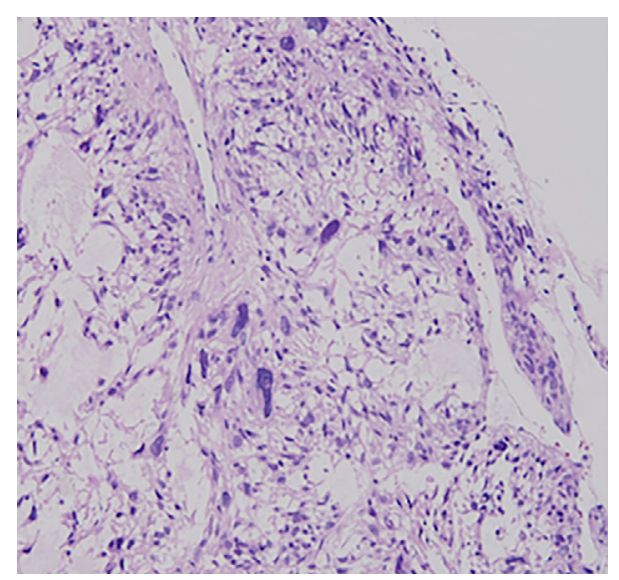

Figure 3. Hematoxylin and Eosin staining of a biopsy specimen of the substance in the superficial femoral vein revealed that it was composed of eosinophilic spindle cells with atypia (Magnification $\times \mathbf{2 0 0}$ ). switched to second-line therapy with eribulin because of the risk of doxorubicin-induced cardiotoxicity. The masses were significantly diminished without additional radiation or resection therapy (Fig. 4). She survived for $>2$ years with chemotherapy alone.

\section{Discussion}

There were two important clinical findings in the present case. First, the measurement of D-dimer helped us to exclude the possibility that the patient's PE was caused by DVT. The patient had a Well's score for PE of 7.5 points, which was enough for a diagnosis of PE due to DVT; however, tests for D-dimer were negative at all stages. At this point, we reconsidered the possibility that other diseases could have caused the pulmonary artery and deep vein defects. Lesions that can occur in the deep veins include old deep venous thrombosis, bacterial masses, and various tumors. The causes of thrombosis include conditions associated with a hypercoagulable state (i.e., infection, other inflammation, and blood or solid cancers) and blood flow disturbance due to conditions such as heart failure and deep vein thrombosis. Moreover, blood vessel tumors can occur due to blood vessel invasion or can be derived from the vascular endothelium as mesodermal tumors (i.e., hemangiopericytoma, intravascular lymphomatosis, and sarcoma,
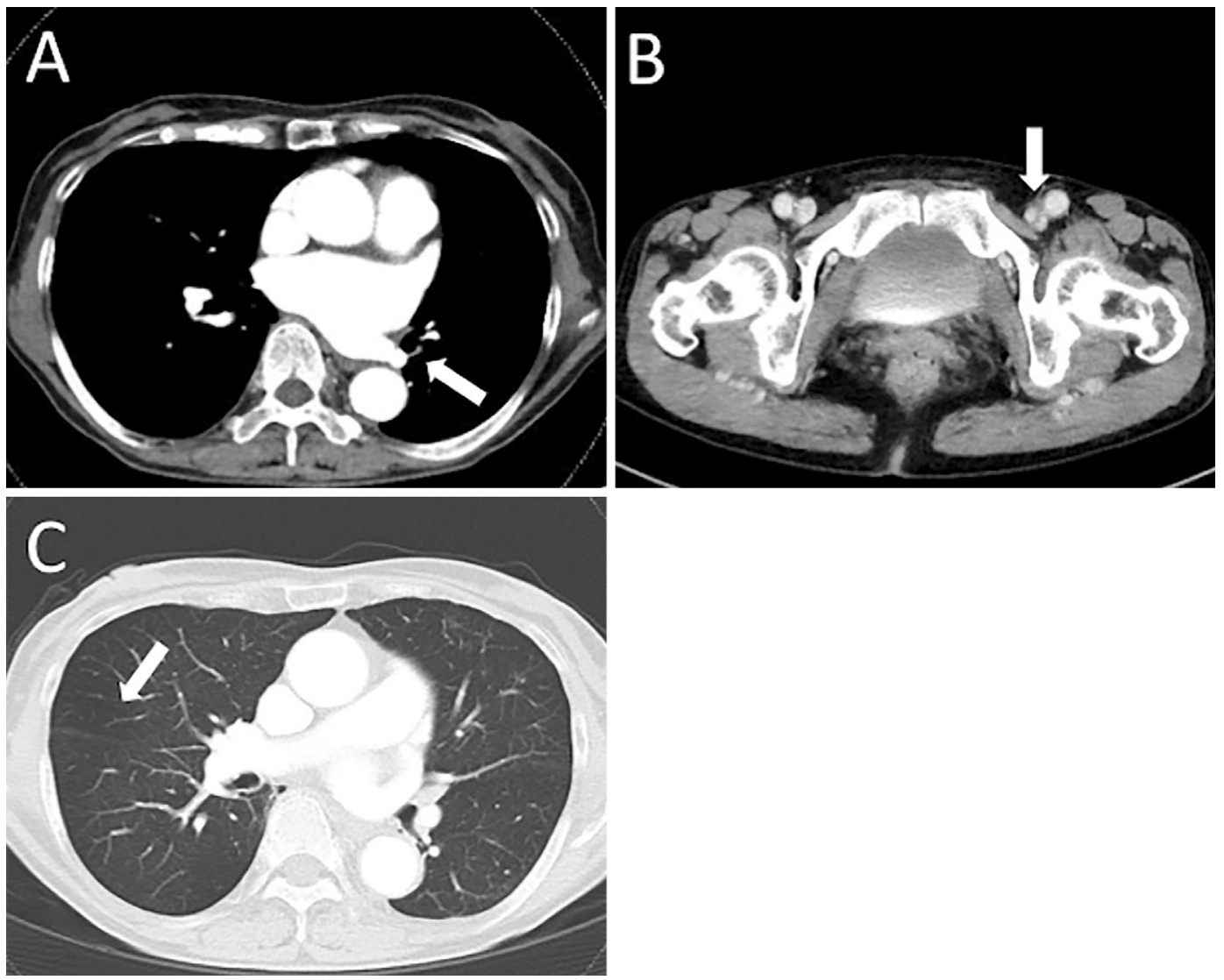

Figure 4. The CT findings at 2 years after chemotherapy: The masses in the deep vein of the left lower limb were diminished $(11.2 \times 14.7 \mathrm{~mm}$; $\mathrm{B}$, arrow) and those in the pulmonary artery and right lung had disappeared (A and $\mathrm{C}$, arrow). 
including vasosarcoma, lymphagiosarcoma, liposarcoma, rhabdomyosarcoma, and leiomyosarcoma) (5). In the current case, we suspected the existence of a tumor because the masses were detected in different vessels, and a new mass was found to have developed in the right lung. A definite diagnosis was made by immunohistochemical staining. Alternatively, thromboembolism may have occurred due to the clot in the leiomyosarcoma. Low D-dimer levels do not completely rule out the possibility of thromboembolism because emboli are generally small. In the current case, the enlargement of the mass despite the administration of anticoagulant therapy for 4 months led to the exclusion of thromboembolism.

Second, leiomyosarcoma can present as an intravenous tumor of the deep veins. Leiomyosarcomas are generally located in the uterus, retroperitoneum, deep soft tissue, or subcutaneous tissue (6); few cases arise from blood vessels. Only $5 \%$ of all reported leiomyosarcoma cases were of vascular origin (6). Among the reported cases of vascular leiomyosarcoma, 40 were located in the iliac vein (7), while 8 were located in the superficial femoral vein (8). Among these 48 cases, there were no cases of PE due to leiomyosarcoma. Only two previous cases described leiomyosarcoma with PE (9); however, the tumors in these cases arose from the deep soft tissue. Leiomyosarcoma of pulmonary vascular origin has recently been reported (10). However, we report the first case of $\mathrm{PE}$ caused by distant intravenous leiomyosarcoma. Many cases of PE caused by leiomyosarcoma may have been overlooked. Leiomyosarcoma of the lower limb can mimic DVT, which can substantially delay its diagnosis (9). Despite being diagnosed with clinical stage IV leiomyosarcoma (with only one lung tumor), the patient survived for a relatively long time because of the small size of the tumor and early detection.

In conclusion, we should consider the possibility of tumors such as leiomyosarcoma when a patient diagnosed with VTE displays atypical progress, especially when a patient's D-dimer level remains low. The consideration of the possibility of leiomyosarcoma in the differential diagnosis and the measurement of the D-dimer level helped in making an early diagnosis of this fatal tumor.
The authors state that they have no Conflict of Interest (COI).

\section{Acknowledgement}

We thank Yasunori Watanabe (Department of Cardiovascular Surgery, Hitachi General Hospital), Tosio Fukusato (Department of Pathology Laboratories, Kotobiken Medical Laboratories), and Hitoaki Saito (Department of Pathology, Ibaraki Prefectural Central Hospital) for helping us with the diagnosis, and Shingo Ishiguro (Department of Clinical Oncology, Ibaraki Prefectural Central Hospital) for providing chemotherapy treatment.

\section{References}

1. Ray P, Birolleau S, Lefort $\mathrm{Y}$, et al. Acute respiratory failure in the elderly: etiology, emergency diagnosis and prognosis. Crit Care 10: R82, 2006.

2. Karande GY, Hedgire SS, Sanchez Y, et al. Advanced imaging in acute and chronic deep vein thrombosis. Cardiovasc Diagn Ther 6: 493-507, 2016.

3. Wells PS, Anderson DR, Rodger M, et al. Derivation of simple clinical model to categorize patients probability of pulmonary embolism: increasing the models utility with the SimpliRED D-dimer. Thromb Haemost 83: 416-420, 2000.

4. Roberts KE, Hamele-Bena D, Saqi A, Stein CA, Cole RP. Pulmonary tumor embolism: a review of the literature. Am J Med 115: 228-232, 2003.

5. Kransdorf MJ. Malignant soft-tissue tumors in a large referral population: distribution of diagnoses by age, sex, and location. AJR Am J Roentgenol 164: 129-134, 1995.

6. Gage MJ, Newman E, Maldonado TS, Hajdu CH. Leiomyosarcoma of the splenic vein. J Vasc Surg 55: 1485-1487, 2012.

7. Fukuda W, Taniguchi S, Fukuda I. Leiomyosarcoma of the external iliac vein. Vascular 20: 178-180, 2012.

8. Yfadopoulos D, Nikolopoulos D, Novi E, Psaroudakis A. Primary superficial femoral vein leiomyosarcoma: report of a case. Surg Today 41: 1649-1654, 2011

9. Perisano C, Maffulli N, Colelli P, Marzetti E, Panni AS, Maccauro G. Misdiagnosis of soft tissue sarcomas of the lower limb associated with deep venous thrombosis: report of two cases and review of the literature. BMC Musculoskelet Disord 14: 64, 2013.

10. Xie $X$, Chen $Y$, Ding $C$, et al. Primary pulmonary leiomyosarcoma: A case report. Oncol Lett 11: 1807-1810, 2016.

The Internal Medicine is an Open Access article distributed under the Creative Commons Attribution-NonCommercial-NoDerivatives 4.0 International License. To view the details of this license, please visit (https://creativecommons.org/licenses/ by-nc-nd/4.0/).

(C) 2018 The Japanese Society of Internal Medicine Intern Med 57: 1425-1428, 2018 